\title{
Optimization of Temperature Drop Formula for Annular Gathering Pipeline in High Water Cut Oil Region of Central Hebei Province
}

\author{
Haonan Cai, Lining Zhao, Xuemei Chen, Xintong Chen, \\ Tong Guan, Jinxiu Wang, Bolin Lv, Shuaibin Li \\ Engineering Technology Institute of PetroChina Huabei Oil Field, Renqiu, China \\ Email:675043739@qq.com
}

How to cite this paper: Cai, H.N., Zhao, L.N., Chen, X.M., Chen, X.T., Guan, T., Wang, J.X., Lv, B.L. and Li, S.B. (2018) Optimization of Temperature Drop Formula for Annular Gathering Pipeline in High Water Cut Oil Region of Central Hebei Province. Journal of Power and Energy Engineering, 6, 21-26. https://doi.org/10.4236/ipee.2018.69003

Received: July 10, 2018 Accepted: September 4, 2018 Published: September 7, 2018

\begin{abstract}
In order to respond to the national policy of energy saving and consumption reduction, the temperature drop formula of annular pipeline should be established to optimize the water mixing temperature and water mixing flow. The traditional temperature drop formula applies the high water cut actual situation to $13.7 \%$. On the basis of the traditional Schulhof temperature drop formula, the influence of hydraulic friction on the oil flow temperature is calculated by the calculus idea. The heat transfer coefficient of the pipeline under the complex condition is obtained by the inverse algorithm, and the water mixing flow and water temperature are simulated with MATLAB. The function surface diagram of the degree and the back station temperature. The actual error can be reduced to $4.8 \%$, and the return station temperature can be optimized by 7 degrees.
\end{abstract}

\section{Keywords}

Circular Water Collecting Oil, Hydraulic Friction, Inverse Algorithm, Saving Energy Cost

\section{Introduction}

East oilfield going most of the oil gradually stepped into the stage of high water cut exploitation (moisture content of $90 \%$ or more), most of the oil collecting process adopted cricoid watered oil gathering process, and the energy consumption of oil collecting part take up little $65 \%-80 \%$ of total energy consumption, for countries to provide energy at the same time, and consumes a lot of energy, as the national energy conservation and consumption reduction policy enforce- 
ment, annular watered oil gathering process saving energy and reducing consumption a difficult task, and in the annular oil gathering process in the past, water temperature and water flow are two key parameters adjusted by production supervisors with artificial experience, now need to through the establishment of theory of pipeline medium temperature drop formula, To calculate the optimal water blending temperature and water blending flow value, so as to achieve the purpose of energy saving and consumption reduction.

However, in the traditional theoretical equation of temperature drop of pipeline media, the schuhof temperature drop formula is usually adopted [1]. Second, in the pipeline buried underground, it is necessary to consider the heat dissipation effect between fluid and pipe wall, wall with thermal insulation layer between the heat dissipation effect, thermal insulation layer and soil between the cooling effect and so on the many kinds of complicated conditions, the total friction coefficient of pipe in the traditional engineering manual already does not apply to the complicated working condition.

\section{Mathematical Model}

In the process of annular oil collection, the change of heat is mainly reflected in the two processes of pipeline heat dissipation and single well fluid mixing during linear transmission [2]. Straight pipes of heat mainly adopts the revised ShuHuoFu temperature drop formula, and in the process of single well output liquid mixture, were it not for the heat loss by the collision, the temperature of the oil pipeline is the starting point should be for a period of piping within the medium mixed with oil well produced fluid temperature, after finishing the conclusion:

$$
T_{j}=\frac{C_{W} G_{W j} T_{W}+\sum_{j=1}^{n} G_{y j} C_{y j} T_{y}}{C_{j}\left(G_{W j}+\sum_{i=1}^{n} G_{y j}\right)}
$$

For the heat capacity of the mixed liquid of $\mathrm{J} /\left(\mathrm{kg} \cdot{ }^{\circ} \mathrm{C}\right)$, for mixed fluid flow $\mathrm{kg} / \mathrm{s}$, as the temperature of the mixed liquid ${ }^{\circ} \mathrm{C}$, for the well produced fluid flow $\mathrm{kg} / \mathrm{s}$, as well the heat capacity of the produced fluid $\mathrm{J} /\left(\mathrm{kg} \cdot{ }^{\circ} \mathrm{C}\right)$, for the mouth of the well produced fluid temperature ${ }^{\circ} \mathrm{C}$.

\subsection{Modify the Schuhof Temperature Drop Formula}

In the process of transmission line, the traditional model using ShuHuoFu temperature drop formula, namely from the starting point of the fluid temperature $L$, starting point for the temperature, the temperature of the surrounding medium for $D \mathrm{~m}$ pipe diameter, pipe length $L, \mathrm{~m}$, pipe quality of oil-water mixture flow $G \mathrm{~kg} / \mathrm{s}$, pipeline total heat transfer coefficient $\mathrm{KW} /\left(\mathrm{m}^{2} \cdot{ }^{\circ} \mathrm{C}\right)$.

$$
T_{L}=T_{0}+\left(T_{Q}-T_{0}\right) e^{\frac{K \pi D L}{C G}}
$$

But in practice, due to the process of crude oil in pipe flow, with the inner wall of pipeline caused by friction, along the pipeline pressure is reduced, to reduce the pressure will be converted into friction heat and fluid inside the pipeline 
heating [3]. The heat loss caused by pipeline hydraulic friction is not considered in the traditional schuhof temperature drop formula.

Revised ShuHuoFu temperature drop formula, is the premise of considering the friction heat, assuming a pipeline diameter $D, G$ throughput and the temperature of the surrounding medium for, take the oil temperature in $d L$ infinitesimal section is $T$, take for hydraulic grade $I$, crude oil temperature drop for $d T$ produced on the $d L$. Under stable conditions, the heat balance equation of $d L$ segment is:

$$
K D \pi\left(T-T_{0}\right) d L=-G C d T+G g i d L
$$

The left part of the equation above is the heat dissipation from the pipeline to the surrounding medium, and the right part is the sum of heat release from oil flow temperature drop and heat of friction resistance [4]. As $d L$ goes up, $d T$ goes down, so minus sign is introduced [5]. After the above equation is deformed, the sukhoff temperature drop formula considering friction-heat generation can be obtained, that is, the modified sukhoff temperature drop formula is:

$$
\begin{gathered}
T_{L}=\left(T_{0}+b\right)+\left[T_{R}-\left(T_{0}+b\right)\right] e^{-a L} \\
a=\frac{K D \pi}{G C}, \quad b=\frac{g i}{C a}
\end{gathered}
$$

\subsection{Fixed the Total Heat Transfer Coefficient of the Pipeline}

Most of the gathering and transportation pipelines are buried pipelines, whose heat transfer is divided into three parts: heat dissipation between the fluid and the inner wall of the pipeline; Heat conduction between the outer wall of the pipe and the insulating layer or the insulating layer; Heat dissipation between the insulation layer and the surrounding environment. The total heat transfer coefficient of crude oil pipeline can be expressed as:

$$
K=\frac{1}{\frac{D}{\alpha_{1} D_{1}}+\sum \frac{D}{2 \gamma_{I}} \ln \frac{D_{(i+1)}}{D_{i}}+\frac{D}{\alpha_{2} D_{w}}}
$$

where $K$ is the total heat transfer coefficient of the pipeline $\mathrm{W} /\left(\mathrm{m}^{2} \cdot{ }^{\circ} \mathrm{C}\right) ; \alpha_{1}$, the heat release coefficient $\mathrm{W} /\left(\mathrm{m}^{2} \cdot{ }^{\circ} \mathrm{C}\right), \alpha_{2}$, between oil flow and pipe inner wall, and is the heat release coefficient $\mathrm{W} /\left(\mathrm{m}^{2} \cdot{ }^{\circ} \mathrm{C}\right) \gamma_{I}$, between pipe wall and soil. Is the heat conduction coefficient $\mathrm{W} /\left(\mathrm{m}^{2} \cdot{ }^{\circ} \mathrm{C}\right)$ corresponding to each layer, and $D$ is the calculated pipe diameter. If there is no insulation layer, the pipe outer diameter can be directly adopted. If there is an insulating layer, the inner diameter of the insulating layer $\mathrm{m}$ is adopted. $D$ is the inner and outer diameter $\mathrm{m}$ of steel tube and asphalt insulation layer; Is the pipe outer diameter $\mathrm{m}$.

However, in the actual production process, due to complex field conditions, the heat transfer coefficient $K$ calculated by the above formula has a large error compared with the actual heat transfer coefficient [6]. In order to reduce this error, the inverse algorithm is usually used to calculate the heat transfer coefficient $K$ of the pipeline. 
$T_{R}$ as the section temperature ${ }^{\circ} \mathrm{C}, T_{Z}$ as section temperature ${ }^{\circ} \mathrm{C}, L_{R}$ for the section length $\mathrm{m}$.

$$
K=\frac{G C}{D L_{R} \pi} \ln \frac{T_{R}-T_{0}}{T_{Z}-T_{0}}
$$

\section{Model Validation and Analysis}

In order to verify the accuracy of the models and applied, the use of Huabei oilfield third production plant is high moisture content of the annular watered oil collecting data (month 12, water temperature, water flow rate, back to the station temperature, pipe diameter size, etc.), and the simulation of temperature drop data and compare [7]. First, use Pipesim to draw the well location diagram, as shown in Figure 1.

According to the ring of the outbound parameters such as temperature, the temperature of back to the station, the inverse calculation formula to calculate the total heat transfer coefficient $\mathrm{K}$, each output of the well fluid flow, temperature, pipe parameters, buried temperature constant coefficient substitution model, the water flow rate set for $\mathrm{x} G$, water blending temperature $\mathrm{T}$ is set to $\mathrm{y}$, back to the station temperature as the dependent variable is set to $\mathrm{z}$. After the mesh treatment of the model with matlab, the relationship between the return station temperature and water incorporation rate and water incorporation temperature is obtained, as shown in Figure 2.

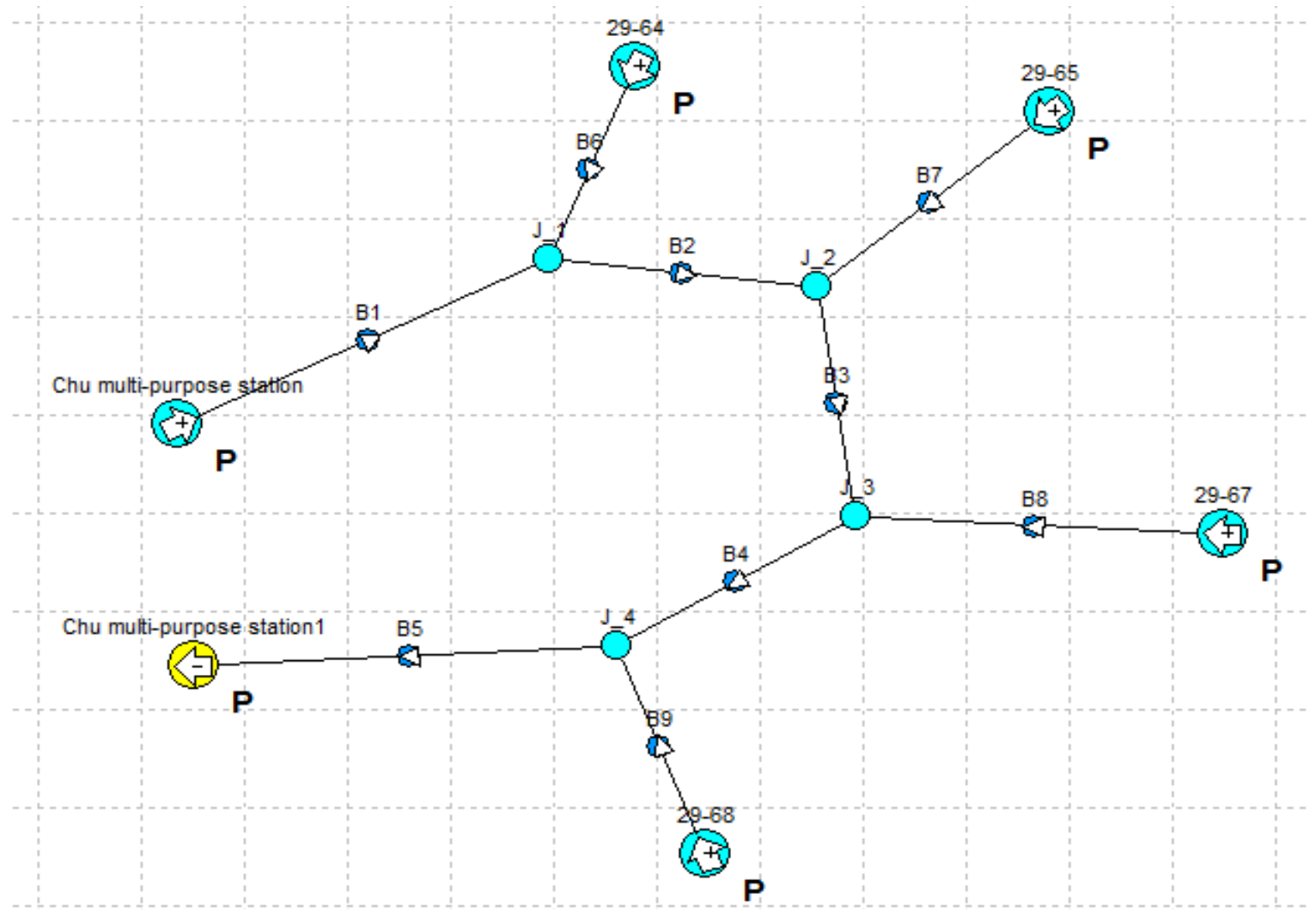

Figure 1. The location map of the east ring of the factory. 
The actual water temperature, water flow into the traditional model (using the traditional ShuHuoFu total heat transfer coefficient of the model and the direct use of engineering manual) and the revised model respectively to calculate the temperature, back to the station to get the following Table 1; The actual temperature of the return station was marked on the graph of the function surface of water incorporation rate, water incorporation temperature and temperature of the return station, as shown in Figure 3.

According to the data obtained, the error between the calculated results of the traditional model and the actual return station temperature is approximately $13.7 \%$. The error between the calculated results of the modified model and the actual return temperature is approximately $4.8 \%$. It can be seen that the calculated

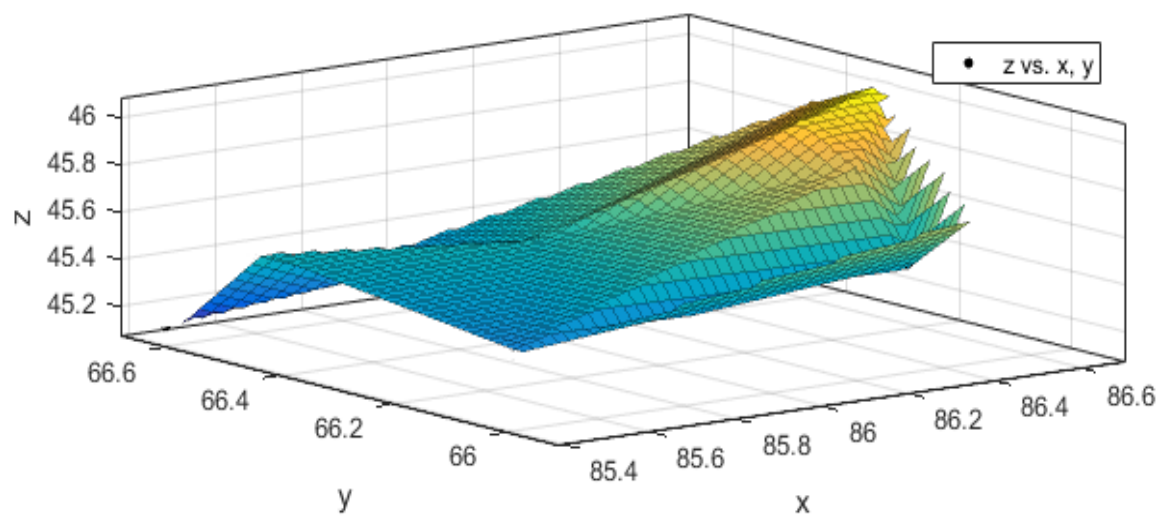

Figure 2. The relation between the temperature of return station and the flow rate and surface function of water blending temperature.

Table 1. Water incorporation rate, water incorporation temperature statistics table.

\begin{tabular}{cccccc}
\hline Date & $\begin{array}{c}\text { Water } \\
\text { flow } \\
\mathrm{m}^{3} / \mathrm{d}\end{array}$ & $\begin{array}{c}\text { The water } \\
\text { temperature } \\
{ }^{\circ} \mathrm{C}\end{array}$ & $\begin{array}{c}\text { Initial return } \\
\text { temperature } \\
{ }^{\circ} \mathrm{C}\end{array}$ & $\begin{array}{c}\text { Traditional model } \\
\text { calculation } \\
{ }^{\circ} \mathrm{C}\end{array}$ & $\begin{array}{c}\text { New model } \\
\text { calculation } \\
{ }^{\circ} \mathrm{C}\end{array}$ \\
\hline 1 & 85.44 & 66.64 & 45.12 & 51.23 & 47.23 \\
2 & 85.44 & 66.49 & 45.52 & 53.26 & 46.89 \\
3 & 85.92 & 66.35 & 45.54 & 50.12 & 47.68 \\
4 & 85.44 & 66.03 & 45.37 & 52.21 & 47.23 \\
5 & 86.4 & 66.06 & 45.46 & 52.33 & 48.13 \\
6 & 86.4 & 65.94 & 45.75 & 53.11 & 48.47 \\
7 & 86.4 & 66.17 & 45.86 & 52.32 & 47.99 \\
8 & 86.64 & 66.29 & 46.04 & 52.11 & 48.24 \\
9 & 86.4 & 66.25 & 45.96 & 53.08 & 48.38 \\
10 & 86.4 & 66.19 & 45.89 & 51.65 & 48.24 \\
11 & 86.4 & 66.15 & 45.83 & 51.22 & 48.13 \\
12 & 86.64 & 66.41 & 45.89 & 51.35 & 48.29 \\
average & 86.16 & 66.2475 & 45.68583 & 51.99917 & 47.90833 \\
\hline
\end{tabular}




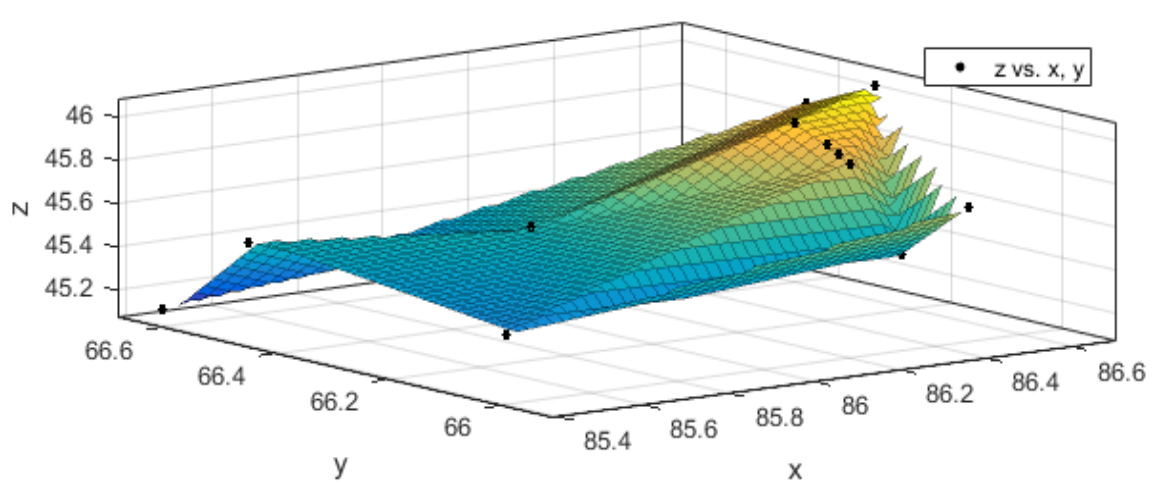

Figure 3. Function surface marked by actual return temperature.

error of the temperature drop formula obtained after the modification is significantly reduced.

\section{Conclusions}

1) After correction ShuHuoFu temperature drop formula and the total heat transfer coefficient, in view of the north China, Huabei Oilfield chu a high water cut oil region, the revised model calculated back to the station temperature compared with traditional model calculated results error is reduced from $13.7 \%$ to $4.8 \%$, the temperature drop of the optimized model for going more in line with high water cut oil ring watered oil temperature drop model, it has application value.

2) Get a more accurate temperature drop model, generally known crude oil freezing point of $35^{\circ} \mathrm{C}$, considering the safety of $3^{\circ} \mathrm{C}$ temperature coefficient, the optimal back stand temperature for $38^{\circ} \mathrm{C}$, and now back to the station temperature $45^{\circ} \mathrm{C}$. In the annular watered oil collecting the existing process, through the model can further adjust the water flow and water temperature, finally can also be made back to the station $7^{\circ} \mathrm{C}$ to $38^{\circ} \mathrm{C}$ temperature drop, so as to further reduce energy consumption.

\section{References}

[1] Su, H. (2017) Optimization of Energy Consumption for Crude Oil Pipeline. Xi'an Petroleum University.

[2] Wu, C.C. and Yan, D.F. (1989) Two Level Hierarchical Optimization Model for Steady Operation of Hot Oil Pipeline. Acta Sinica, 10, 109-117.

[3] Wang, B.Q., Lin, Y.H., Dai, Y.B., et al. (2012) Current Situation and Prospect of China's Crude Oil Pipeline. Petroleum Planning and Design, 23, 8-11.

[4] Liu, Y. and Guan, X.J. (1993) Optimal Design of Oil and Gas Gathering and Transportation System. Natural Gas Industry, No. 2, 36-38.

[5] Liu, Y. and Guan, X.J. (1993) Topology Optimization Design of Annular Gathering Pipeline Network. Acta Petrota Sinica, No. 3, 28-30.

[6] Shi, G.Y. and Dong, J.L. (1999) Optimization Method. Higher Education Press, 53-57.

[7] Jiang, J.S., He, C.X. and Pan, S.H. (2007) Optimization Calculation Method South China University of Technology Press, 63-217. 